Introduction: Gastric cancer (GC) is the fourth most common malignant disease in the world, following breast cancer, colorectal cancer, and lung cancer. This study aimed to evaluate the usefulness of multidetector-row computed tomography (MDCT) in identifying the metastatic lymph node of GC.

Material and methods: A cross-sectional study was performed after receiving approval by the institutional review board. A total of 88 patients with GC, who underwent radical gastrectomy, were examined by MDCT. Categorical variables were compared using Fisher's exact test. The discriminating ability of lymph node size was determined according to an area under the receiver operating curve (AUROC) analysis, and the optimal cut-off point was determined.

Results: The proportion of metastatic lymph node patients in the proximal group (32.3\%) was significantly higher than that in the distal group (18.4\%). $T$ categorisation and lymph node sizes were significantly different between the nonmetastatic lymph node and metastatic lymph node groups. The AUROC for lymph node size was 0.738 , with an optimal cut-off point of $7.5 \mathrm{~mm}$, producing a sensitivity of $71.5 \%$ and a specificity of $70.5 \%$.

Conclusions: MDCT displayed medium accuracy for the determination of metastatic lymph nodes and $\mathrm{N}$ categorisation. Based on our findings, although MDCT is generally the first choice for preoperative assessments in GC patients, other diagnostic modalities should supplement MDCT in order to achieve more precise $\mathrm{N}$ staging.

Key words: MDCT, gastric cancer, lymph node, $\mathrm{N}$ staging.

Contemp Oncol (Pozn) 24 (2): 125-131 DOI: https://doi.org/10.5114/wo.2020.97484

\section{The value of multidetector-row computed tomography in lymph node staging of gastric cancer: a preliminary Vietnamese study}

\author{
Nguyen Van Sang ${ }^{1}$, Nguyen Minh Duc ${ }^{2,3}$, Pham Hong Duc ${ }^{4}$, Phung Anh Tuan ${ }^{5}$ \\ ${ }^{1}$ Department of Radiology, Hanoi University of Public Health, Hanoi, Vietnam \\ ${ }^{2}$ Department of Radiology, Pham Ngoc Thach University of Medicine, Ho Chi Minh City, \\ Vietnam \\ ${ }^{3}$ Department of Radiology, Children's Hospital 2, Ho Chi Minh City, Vietnam \\ ${ }^{4}$ Department of Radiology, Hanoi Medical University, Hanoi, Vietnam \\ ${ }^{5}$ Department of Radiology, Vietnam Military Medical University, Hanoi, Vietnam
}

\section{Introduction}

Gastric cancer (GC) is the fourth most common malignant disease in the world, following breast cancer, colorectal cancer, and lung cancer [1]. Although the incidence of GC has been decreasing, it remains the most prevalent form of cancer in Asian countries, including Vietnam [1, 2]. Radical surgical resection is a common curative treatment for GC. However, the mortality rate remains high, with a five-year survival rate of approximately 30-35\% [3]. Surgical results depend on the GC stage at presentation, which includes the depth of tumour invasion and the extent of local lymph node and distant metastases. Therefore, determining the exact GC stage before surgery is essential for establishing suitable treatment plans. Multidetector-row computed tomography (MDCT) is the most commonly used modality for preoperative GC assessments. MDCT image can be usefully employed to determine tumour size and location ( $T$ staging) and to identify distant organ metastasis. However, the role of MDCT during the identification and categorisation of cancerous lymph nodes ( $\mathrm{N}$ staging) remains controversial $[4,5]$. The present study aimed to assess the role of MDCT in the staging and classification of lymph nodes during GC.

\section{Material and methods}

Study design and participants

A cross-sectional study of 88 patients was conducted in Hanoi, Vietnam, from September 2015 to October 2016. The participants were GC patients who had undergone radical gastrectomy at 108 Military Central Hospital in Hanoi. The inclusion criteria were as follows: 1) patients diagnosed with GC by gastroscopic biopsy; 2) patients naive to neoadjuvant chemo-radio-therapeutic treatment before surgery; and 3) patients with no history of allergy to iodine contrast agents. The exclusion criteria were as follows: 1) presenting with other cancers, in addition to GC; and/or 2) recurrent GC. All participants underwent MDCT examinations, using a unique protocol. The imaging findings were compared with histopathological results.

This study was approved by the Scientific Committee in Biomedical Research, 108 Military Central Hospital (Ref: 262/QĐ-V108, dated 10 Sep 2015). All patients in the study provided informed consent before collecting data, and all were informed of their complete right to withdraw from the study at any time, without threat or disadvantage. 


\section{Data collection}

Data collection was performed by two experienced radiologists, who are the authors of the present manuscript. Data regarding the demographic and clinical characteristics of the patients were extracted from the participants' medical records. The MDCT images were collected according to a unique protocol template.

\section{MDCT protocol}

MDCT was performed using a 16-detector-row computed tomography (CT) scanner (BRIVO CT 385, GE Health care, USA). The parameters were as follows: slice thickness $1 \mathrm{~mm}$, rotation time 1 second, and pitch 1.75, $120 \mathrm{kVp}$, and $250 \mathrm{mAs}$. All patients were examined after fasting for at least eight hours and received approximately 500-1000 ml of warm water, orally, prior to the study. An intramuscular injection of $20 \mathrm{mg}$ hyoscine butylbromide (Buscopan, Boehringer Ingelheim, Spain) was administered 10 minutes prior to scanning. Then, $100 \mathrm{ml}$ iobitridol (Xenetix 300, Guerbet, France) was injected into the cubital vein, using an automated injector device, at a velocity of $3.0 \mathrm{ml} / \mathrm{s}$. Arterial and venous phase images were acquired at 30 and $70 \mathrm{sec}$ after the contrast injection, respectively [6, 7]. All patients were in a supine position, and the scan range was extended from the diaphragm to the pubic symphysis. After scanning, reconstructions were performed using multiplanar reformations (Fig. 1)

\section{MDCT image analysis}

Two radiologists, with 15 and 20 years of experience and without knowledge of patient information, independently analysed the imaging data. The inter-observer agreement was excellent for all MDCT findings. Thus, the results of the first reader (first author) were used for all further analyses.

The collected lymph node data included the number, location, diameter of the short axis, and enhancement level. Primary tumour data were also recorded. To detect lymph nodes, the 1-mm-thick axial images were examined in cine-mode display. To measure the sizes of lymph nodes, both axial images and oblique multiplanar reformation images were used to identify the longest diameter of each lymph node in all three dimensions. The short diameter of the lymph node was measured perpendicular to the longest axis in the same image. The MDCT nodal groups were defined based on anatomic landmarks, and 16 groups were identified according to the Japanese Research Society for Gastric Cancer (JRSGC) guidelines [8]. Lymph nodes were considered metastatic if the short-axis diameter was greater than $8 \mathrm{~mm}$. N categorisation was determined according to the $7^{\text {th }}$ edition of the American Joint Committee on Cancer (AJCC) tumour, nodes, and metastases (TNM) staging system [9].

\section{Surgery}

All patients underwent radical gastrectomy and lymphadenectomy. The category of lymphadenectomy and the grouping of resected lymph nodes were decided by surgeons, based on the surgical findings. The same nodal grouping system was applied to all lymph nodes, regardless of whether they were detected during surgery and/or by MDCT. The surgeons defined and marked each lymph node detected by MDCT. Then, all resected lymph nodes were examined histopathologically, to confirm metastasis for each nodal group.

\section{Statistical analysis}

Statistical analyses were performed using SPSS version 22 (IBM Corp., New York, USA). Categorical variables were compared using Fisher's exact test. The accuracy of MDCT for $\mathrm{N}$ categorisation was calculated by comparing the images with the histopathological findings. The ability to use lymph node size to determine metastasis was evaluated based on the area under the receiver operating characteristic curve (AUROC) analysis. The optimal cut-off point was identified based on the Youden index, which was then
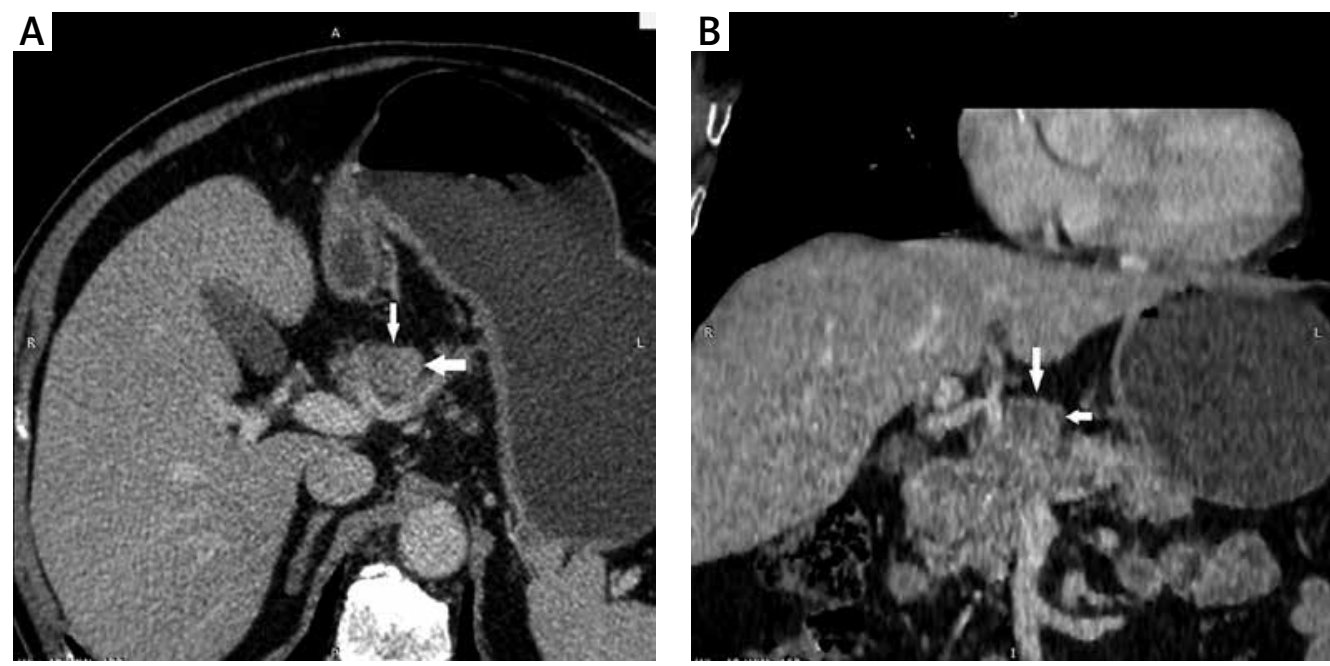

Fig. 1. Images of a 78-year-old man with well-differentiated adenocarcinoma, T3 stage. (A) Axial MDCT image and (B) reconstructed coronal MDCT image. The white arrows indicate the group 8 lymph nodes. The short diameter of the lymph node was $26 \mathrm{~mm}$. This was proven metastatic lymph node by histopathological examination 
Table 1. Numbers of lymph nodes detected by multidetector-row computed tomography (MDCT) and histopathological examinations, according to nodal group

\begin{tabular}{|c|c|c|c|c|c|c|}
\hline \multirow[t]{2}{*}{ Nodal group } & & \multicolumn{2}{|c|}{ Histopathological examination } & \multicolumn{3}{|c|}{ MDCT examination } \\
\hline & & $\begin{array}{c}\text { Number of nodes } \\
\text { detected }\end{array}$ & $\begin{array}{c}\text { Number } \\
\text { of metastatic nodes }\end{array}$ & $\begin{array}{c}\text { Number of nodes } \\
\text { detected }\end{array}$ & $\begin{array}{l}\text { Metastatic nodes } \\
\text { MDCT + }\end{array}$ & $\begin{array}{l}\text { Metastatic nodes } \\
\text { histopathology + }\end{array}$ \\
\hline \multirow{7}{*}{$\begin{array}{l}\text { Proximal } \\
\text { group }\end{array}$} & 1 & 4 & $0(0)$ & $2(50)$ & 1 & $0(0)$ \\
\hline & 2 & 9 & $3(33.3)$ & $1(11.1)$ & 0 & $0(0)$ \\
\hline & 3 & 454 & $142(31.3)$ & $207(45.6)$ & 89 & $82(39.6)$ \\
\hline & 4 & 120 & $20(16.7)$ & $50(41.7)$ & 16 & $8(16)$ \\
\hline & 5 & 54 & $22(40.7)$ & $30(55.6)$ & 18 & $12(40)$ \\
\hline & 6 & 349 & $133(38.1)$ & $133(38.1)$ & 58 & $53(39.8)$ \\
\hline & $1-6$ & 990 & $320(32.3)^{\mathrm{a}}$ & $423(42.7)^{b}$ & 182 & $155(36.6)^{c}$ \\
\hline \multirow[t]{11}{*}{ Distal group } & 7 & 8 & $1(12.5)$ & $2(25)$ & 1 & $0(0)$ \\
\hline & 8 & 38 & $7(18.4)$ & $18(47.4)$ & 10 & $5(27.8)$ \\
\hline & 9 & 7 & $5(71.4)$ & $3(42.9)$ & 3 & $2(66.7)$ \\
\hline & 10 & 0 & 0 & 0 & 0 & 0 \\
\hline & 11 & 0 & 0 & 0 & 0 & 0 \\
\hline & 12 & 21 & $5(23.8)$ & $3(14.3)$ & 3 & $2(66.7)$ \\
\hline & 13 & 35 & $3(8.6)$ & $10(28.6)$ & 7 & $1(10)$ \\
\hline & 14 & 5 & $0(0)$ & $1(20)$ & 0 & $0(0)$ \\
\hline & 15 & 0 & 0 & 0 & 0 & 0 \\
\hline & 16 & 0 & 0 & 0 & 0 & 0 \\
\hline & $7-16$ & 114 & $21(18.4)^{a}$ & $37(32.5)^{b}$ & 24 & $10(27)^{c}$ \\
\hline Total & & 1104 & $341(30.9)$ & $460(41.7)$ & 206 & $165(35.9)$ \\
\hline
\end{tabular}

used to calculate the sensitivity, specificity, and accuracy of MDCT-based diagnoses. A p-value of $<0.05$ was considered significant for all statistical analyses.

\section{Results}

According to Table 1, of the 1104 lymph nodes that were resected and examined histopathologically, 341 nodes (30.9\%) were positive for metastasis. At MDCT, 464 nodes were detected. Of these, four nodes were determined histopathologically to be false positives and were excluded from the study. Of the 460 lymph nodes detected at MDCT, 206 nodes were considered metastatic nodes (size $\geq 8 \mathrm{~mm}$ ). However, only 165 of these nodes were determined to be metastatic based on the histopathological analysis. The mean short-axis diameter of metastatic lymph nodes (10.10 $\pm 5.31 \mathrm{~mm}$; range $4-35 \mathrm{~mm})$ was larger than that of non-metastatic lymph nodes $(6.89 \pm 2.12 \mathrm{~mm}$; $4-17 \mathrm{~mm}$, $p<0.001$.

Nodal group analysis showed that 990 lymph nodes were in the proximal group and 114 nodes were in the distal group. The proportion of metastatic lymph nodes in the proximal group (32.3\%; 320/990) was significantly higher than that in the distal group (18.4\%; 21/114). Approximately $94 \%$ of total metastatic lymph nodes were determined to be located in the proximal group, although both benign lymph nodes and metastatic lymph nodes were founded in all six nodal groups included in the proximal group. In contrast, metastatic lymph nodes were not founded in nodal groups $10,11,14,15$, and 16 , in the distal group.

Of the 460 lymph nodes detected by MDCT, approximately 92\% (423 nodes) were identified in the proximal group. The proportion of lymph nodes detected by MDCT in the proximal group was also higher than that in the distal group (42.7\% vs. $32.5 \%, p<0.05)$. Although the total number of metastatic lymph nodes identified in the proximal group was higher than that in the distal group, no significant difference in the percentage of identified metastatic nodes was found between groups (36.6\% vs. $27 \%$, $p>0.05)$.

Table 2 shows the numbers of resected lymph nodes, metastatic lymph nodes, and lymph nodes detected by MDCT, according to size. The majority of resected lymph nodes $(72.3 \%)$ were small $(<8 \mathrm{~mm})$. The proportion of MDCT-identified metastatic lymph nodes and the sensitivity with which MDCT detected lymph nodes increased as node sizes increased. Of 798 resected lymph nodes < $8 \mathrm{~mm}$, only 193 nodes (24.5\%) were determined to be metastatic, whereas 148 of 306 nodes $(48.4 \%) \geq 8 \mathrm{~mm}$ were determined to be metastatic, which represented a significant difference. The ability of MDCT to identify nodes $<8 \mathrm{~mm}$ (32.2\%) was significantly reduced compared to that for nodes $\geq 8 \mathrm{~mm}$ (67.3\%). Similarly, the proportion of metastatic lymph nodes $<8 \mathrm{~mm}(18.5 \%)$ detected by MDCT was significantly lower than the proportion $\geq 8 \mathrm{~mm}$ 
Table 2. Numbers of lymph nodes detected by multidetector-row computed tomography (MDCT) and histopathological examination, according to sizes

\begin{tabular}{|c|c|c|c|c|}
\hline \multirow{2}{*}{$\begin{array}{l}\text { Size } \\
(\mathrm{mm})\end{array}$} & \multicolumn{2}{|c|}{ Histopathological examination } & \multicolumn{2}{|c|}{ MDCT examination } \\
\hline & Number of nodes detected & Number of metastatic nodes ${ }^{a}$ & Number of nodes detected ${ }^{b}$ & Number of metastatic nodes ${ }^{c}$ \\
\hline 1 & 4 & $0(0)$ & $0(0)$ & 0 \\
\hline 2 & 48 & $5(10.4)$ & $0(0)$ & 0 \\
\hline 3 & 161 & $22(13.7)$ & $0(0)$ & 0 \\
\hline 4 & 162 & 28 (17.3) & $10(6.2)$ & $1(10)$ \\
\hline 5 & 251 & 79 (31.5) & 99 (39.8) & $19(19.2)$ \\
\hline 6 & 98 & $37(37.8)$ & $88(89.8)$ & $19(21.6)$ \\
\hline 7 & 74 & $22(29.7)$ & $57(77.0)$ & $8(14)$ \\
\hline $1-7$ & 798 & $193(24.5)$ & $254(32.2)$ & 47 (18.5) \\
\hline 8 & 67 & $29(43.3)$ & $58(86.6)$ & $27(46.6)$ \\
\hline 9 & 27 & $14(51.9)$ & $21(77.8)$ & $8(38.1)$ \\
\hline 10 & 119 & $46(38.7)$ & $76(63.9)$ & $45(59.2)$ \\
\hline 11 & 15 & $7(46.7)$ & $7(46.7)$ & $2(28.6)$ \\
\hline 12 & 28 & 15 (53.6) & $14(50.0)$ & $12(85.7)$ \\
\hline 13 & 2 & $2(100)$ & $0(0)$ & 0 \\
\hline 14 & 7 & $5(71.4)$ & $5(71.4)$ & $4(80)$ \\
\hline 15 & 20 & $13(65.0)$ & $6(30.0)$ & $4(66.7)$ \\
\hline 16 & 3 & $2(66.7)$ & 3 (100) & $2(66.7)$ \\
\hline 17 & 1 & $1(100)$ & $1(100)$ & $0(0)$ \\
\hline 18 & 0 & $0(0)$ & $0(0)$ & 0 \\
\hline 19 & 0 & $0(0)$ & $0(0)$ & 0 \\
\hline 20 & 8 & $5(62.5)$ & $5(62.5)$ & 5 (100) \\
\hline$>20$ & 9 & 9 (100) & 9 (100) & 9 (100) \\
\hline$\geq 8$ & 306 & $148(48.4)$ & $206(67.3)$ & 118 (57.3) \\
\hline Total & 1104 & 341 (30.9) & $460(41.7)$ & 165 (35.9) \\
\hline
\end{tabular}

(57.3\%). All lymph nodes > $20 \mathrm{~mm}$ were metastatic and correctly identified by MDCT.

The value of MDCT for N staging is shown in Table 3. The sensitivity, specificity, and accuracy were NO: 51.4\%, 90.6\%, and 75\%; N1: 81.3\%, 75\%, and 76.1\%; N2: 58.8\%, 77.5\%, and $73.9 \%$; and N3: $40 \%, 100 \%$, and $86.4 \%$, respectively. The overall accuracy of MDCT for N staging was $55.7 \%$. The consistency between MDCT imaging and the postsurgical histopathologic results for $\mathrm{N}$ staging was medium, with a $\kappa$ value of 0.521 (95\% confidence interval [Cl]: 0.393-0.649). MDCT both overestimated and underestimated $\mathrm{N}$ staging assessments, with $20.4 \%$ of assessments representing overestimates and $23.9 \%$ representing underestimates.

Table 4 shows the relationships between patients' characteristics and MDCT findings, according to N staging results. $T$ staging results and lymph nodes size showed significant differences between the non-metastatic and metastatic lymph node groups. Of 14 patients in the T1 category, 13 patients were classified as NO and one patient was N2. Of the 16 patients in the T2 category, the number of patients classified as N0, N1, N2, and N3 were 8, 4,2 , and 2, respectively. Of 58 patients in the T3 category, the number of patients classified as NO, N1, N2, and N3 were $14,12,14$, and 18 , respectively. These results showed that as tumour infiltration progressed, the ability to detect lymph node metastasis increased.

Figure 2 shows the AUROC results for the ability of lymph node size to discriminate between metastatic and non-metastatic lymph nodes, with an AUROC value of 0.738 and an optimal cut-off point of $7.5 \mathrm{~mm}$. When applying this cut-off point, the sensitivity and specificity were $71.5 \%$ and $70.5 \%$, respectively.

\section{Discussion}

Lymph node metastasis is among the most important prognostic factors in GC. The five-year survival rate of patients without lymph node metastasis can reach as high as $85-90 \%$ [5]. MDCT is the most common technique used for the preoperative determination of $\mathrm{N}$ categories in $\mathrm{GC}$ patients. However, the diagnostic abilities of MDCT have varied among studies conducted in different countries [10]. In the present study, the proportion of metastatic lymph nodes in the proximal group was higher than that in the distal group. The proportion of lymph nodes in the 
Table 3. $\mathrm{N}$ categorisation of gastric cancers, by multidetector-row computed tomography (MDCT) and histopathological examinations

\begin{tabular}{lccccc}
\multirow{2}{*}{$\begin{array}{l}\text { MDCT } \\
\text { categorisation }\end{array}$} & \multicolumn{3}{c}{$\begin{array}{c}\text { Histopathological } \\
\text { categorisation }\end{array}$} & $p$-value \\
\cline { 2 - 5 } & N0 & N1 & N2 & N3 & \\
N0 & 18 & 2 & 3 & 0 & $<0.001$ \\
N1 & 11 & 13 & 4 & 3 & \\
N2 & 6 & 1 & 10 & 9 & \\
N3 & 0 & 0 & 0 & 8 &
\end{tabular}

proximal group detected by MDCT was also higher than that in the distal group. Fukuya et al. found that the MDCT sensitivities for the detection of nodes in groups 3 and 5 were significantly higher and lower, respectively, relative to the overall sensitivity for the detection of lymph nodes, and none of the nodes in nodal group 12 were detected [11]. Jiang et al. found that the accuracies of MDCT for the diagnosis of metastatic lymph nodes in groups 2,10 , and 13 reached $98.9 \%$, and the sensitivities for groups 2, 9, 10, and 13 were $100 \%$ [7]. In contrast, Kim et al. reported that MDCT showed relatively low accuracy and positive predictive values for the perigastric lymph nodes near the lesser or greater curvature (groups 3 and 4) but demonstrated higher accuracy and positive predictive values for the lymph node groups 1, 5, 6, 7, 8, 9, and 11 [6].

We found that the short-axis diameters of metastatic lymph nodes were larger than those of non-metastatic lymph nodes. The proportion of metastatic lymph nodes and the sensitivity of MDCT for the detection of lymph nodes increased as the node size increased. In a previous study by Fukuya et al., lymph nodes were positive for metastasis in $5.1 \%$ of nodes $<5 \mathrm{~mm}, 21.7 \%$ of nodes $5-9 \mathrm{~mm}$, $23 \%$ of nodes $10-14 \mathrm{~mm}$, and $82.6 \%$ of nodes $>14 \mathrm{~mm}$ [11]. Of 382 MDCT-detected lymph nodes, Morgagni et al. found that only $22.9 \%$ of 261 lymph nodes that were not radiologically detected were infiltrated, including $16 \%$ of nodes $<5 \mathrm{~mm}, 33 \%$ of nodes between 5 and $10 \mathrm{~mm}$, and 63\% of nodes between 15 and $20 \mathrm{~mm}$ [12]. Kim and Kim also revealed that the sizes of metastatic lymph nodes were significantly larger than those of non-metastatic lymph nodes. As lymph node sizes increased, the proportion of metastatic lymph nodes out of all detected lymph nodes increased [13]. All results showed that size was an important criterion for determining metastasis and could be used for differentiation by MDCT. In this study, the lymph nodes were detected by both axial and oblique multiplanar reformation images. We believe that these techniques are the best for achieving precise measurements and represent a meaningful contribution to the field.

The reported accuracy of MDCT has varied among previous studies. Jiang et al. reported an overall accuracy of $86.3 \%$ for N staging based on MDCT findings, and the consistency between MDCT images and pathological N staging results (except for $\mathrm{N} 2$ ) was good, with $\kappa$ values ranging from 0.449 to 0.662 [7]. In a study by Yan et al., the sensitivity and specificity of MDCT for the preoperative prediction of metastatic lymph nodes in GC were $77.4 \%$ and $73.3 \%$, respectively, whereas the sensitivity and specificity of MDCT for the
Table 4. Patient characteristics and multidetector-row computed tomography (MDCT) findings relative to $\mathrm{N}$ category

\begin{tabular}{|c|c|c|c|c|c|}
\hline \multirow[t]{2}{*}{ Characteristics ( $n$ ) } & \multicolumn{4}{|c|}{$\mathrm{N}$ category } & \multirow[t]{2}{*}{$p$-value } \\
\hline & NO & N1 & N2 & N3 & \\
\hline Sex & & & & & 0.074 \\
\hline Male (65) & 25 & 11 & 10 & 19 & \\
\hline Female (23) & 20 & 5 & 7 & 1 & \\
\hline Age & & & & & 0.66 \\
\hline$<40(2)$ & 0 & 1 & 1 & 0 & \\
\hline $40-59(27)$ & 11 & 5 & 6 & 5 & \\
\hline$\geq 60$ (59) & 24 & 10 & 10 & 15 & \\
\hline Tumour location & & & & & 0.52 \\
\hline Upper third (3) & 1 & 0 & 2 & 0 & \\
\hline Middle third (29) & 12 & 4 & 4 & 9 & \\
\hline Lower third (53) & 21 & 11 & 11 & 10 & \\
\hline Total (3) & 1 & 1 & 0 & 1 & \\
\hline Tumour size & & & & & 0.85 \\
\hline$<40 \mathrm{~mm}(49)$ & 21 & 8 & 10 & 10 & \\
\hline$\geq 40$ mm (39) & 14 & 8 & 7 & 10 & \\
\hline T category & & & & & 0.003 \\
\hline T1a (4) & 4 & 0 & 0 & 0 & \\
\hline $\mathrm{T} 1 \mathrm{~b}(10)$ & 9 & 0 & 1 & 0 & \\
\hline T2 (16) & 8 & 4 & 2 & 2 & \\
\hline T3 (58) & 14 & 12 & 14 & 18 & \\
\hline Nodal group & & & & & 0.24 \\
\hline Proximal (423) & 268 & 155 & & & \\
\hline Distal (37) & 27 & 10 & & & \\
\hline Lymph node size & & & & & $<0.001$ \\
\hline$<8(254)$ & 207 & 47 & & & \\
\hline$\geq 8(206)$ & 88 & 118 & & & \\
\hline
\end{tabular}

Bolded p-values indicate significance



Fig. 2. Receiver operating characteristic curve indicates the sensitivity, specificity, and optimal cut-off value of lymph node size for the differentiation of metastatic lymph nodes 
preoperative prediction of N2 and N3 were $15.4 \%$ and $100 \%$, respectively [14]. According to Kim et al., the overall accuracy of N staging by MDCT was $63.2 \%$. Of 106 NO cases, MDCT overestimated 11 cases (10.4\%). Of the 65 cases categorised as N1 or higher, MDCT underestimated 48 cases (73.8\%) and overestimated four cases (6.2\%). Overall, MDCT had a sensitivity of $60.0 \%$, a specificity of $89.6 \%$, an accuracy of $78.4 \%$, and a positive predictive value of $78.0 \%$ for detecting the presence of lymph node metastasis [6]. Luo et al. performed a meta-analysis that included 27 studies and 6519 subjects, to evaluate the value of MDCT for pre-operative lymph node staging [10]. The authors revealed that the value was variable, with sensitivities ranging from $17 \%$, as reported by Ahn et al. [15], to 97\%, as reported by Karakoyun et al. [16], and specificities ranging from $61 \%$, as reported by Feng et al. [17] and Pan et al. [18], to 100\%, as reported by Joo et al. [19]. The meta-analysis reported summary sensitivity, specificity, and AUROC values of $0.67(95 \% \mathrm{Cl}, 0.56-0.77), 0.86(95 \% \mathrm{Cl}$, $0.81-0.90)$, and $0.86(95 \% \mathrm{Cl}, 0.83-0.89)$, respectively, with mild heterogeneities. These results indicated that the ability of MDCT to determine preoperative lymph node staging in GC patients was limited due to low sensitivity

The criteria for defining metastatic lymph nodes using preoperative MDCT differed among previous studies. Most studies defined metastatic lymph nodes based on the short-axis diameter, with nodes considered to be metastatic if the short-axis diameter was larger than $10 \mathrm{~mm}$ [20], 8 $\mathrm{mm}[6,21,22]$, or $6 \mathrm{~mm}$ for perigastric lymph nodes, and larger than $8 \mathrm{~mm}$ for extraperigastric lymph nodes [14, 23]. In addition to size, central necrosis, strong enhancement, and clusters of three or more perilesional nodes were also considered to be indicators of local metastasis. In this study, a short-axis diameter larger than $8 \mathrm{~mm}$ was used to differentiate metastatic lymph nodes, which is similar to the criterion used by most studies. However, central necrosis and/or enhancement are only recognised in larger nodes. In the present study, using size as an independent standard, the AUROC value was 0.738 , with an optimal cutoff point of $7.5 \mathrm{~mm}$, resulting in a sensitivity and specificity of $71.5 \%$ and $70.5 \%$, respectively. These results were similar to those reported by Yan et al. [14] (AUROC: 0.789, cut-off value: $0.585 \mathrm{~cm}$, sensitivity: $80.2 \%$, and specificity: $66.4 \%$ ) and Bai et al. [20] (AUROC: 0.807, cut-off point: $6.0 \mathrm{~mm}$, sensitivity: $75.8 \%$, and specificity: $75.6 \%$ ).

The $\mathrm{N}$ category also depends on tumour infiltration. In this study, only $7.1 \%$ of patients categorised as $\mathrm{T} 1$ had metastatic lymph nodes, whereas $75.9 \%$ of patients categorised as T3 had metastatic lymph nodes. As the tumour aggressiveness increased, the proportion of metastatic lymph nodes increased. Jiang et al. found that $100 \%$ of $\mathrm{T} 1$ patients, $95.7 \%$ of T2 patients, $44.5 \%$ of $\mathrm{T} 3$ patients, and $20.7 \%$ of T4 patients were classified as NO. The N3 classification was only identified in $18.5 \%$ of $\mathrm{T} 3$ patients and $48.3 \%$ of $\mathrm{T} 4$ patients [7]. Kwee et al. reviewed several articles and found that patients with T1 tumours have a low risk of lymph node metastasis [24]. Metastatic lymph nodes were found in $2.2 \%$ of patients classified as T1a and in $17.9 \%$ of patients classified as T1b GC [25]. In patients with T2 and T3 tumours, the proportions who presented with lymph node metastasis reached $44 \%$ and $64 \%$, respectively [2].

This study had some limitations. First, only a small number of patients were evaluated. The results could change in a study examining a larger number of cases. Second, although we attempted to match the lymph nodes resected during surgery with those identified by MDCT, in some cases the surgeons were not able to determine exact matches, which could result in some correlation errors.

\section{Conclusions}

In conclusion, MDCT displayed medium accuracy for the preoperative determination of $\mathrm{N}$ staging and the detection of nodal group metastasis. Based on our findings, although MDCT is the first choice for the preoperative assessment of GC patients, other supplementary diagnostic modalities should be used, to achieve more precise N staging results.

The authors declare no conflict of interest.

\section{References}

1. Torre LA, Bray F, Siegel RL, Ferlay J, Lortet-Tieulent J, Jemal A. Global cancer statistics, 2012. CA Cancer J Clin 2015; 65: 87-108.

2. Hohenberger P, Gretschel S. Gastric cancer. Lancet 2003; 362: 305-15.

3. Chon SH, Berlth F, Plum PS, et al. Gastric cancer treatment in the world: Germany. Transl Gastroenterol Hepatol 2017; 2: 53.

4. Marco M, Amato ASI, Federico C, Arnaldo S, Giuseppe A. The role of computed tomography in the imaging of gastric carcinoma. In: Management of gastric cancer. InTech, Croatia 2011: 3-20.

5. Hallinan JT, Venkatesh SK. Gastric carcinoma: Imaging diagnosis, staging and assessment of treatment response. Cancer Imaging 2013; 13: 212-27.

6. Kim SH, Kim JJ, Lee JS, et al. Preoperative $n$ staging of gastric cancer by stomach protocol computed tomography. J Gastric Cancer 2013; 13: 149-56.

7. Jiang $M$, Wang $X$, Shan $X$, et al. Value of multi-slice spiral computed tomography in the diagnosis of metastatic lymph nodes and n-stage of gastric cancer. J Int Med Res 2019; 47: 281-92.

8. Japanese Gastric Cancer Association. Japanese classification of gastric carcinoma: 3rd english edition. Gastric Cancer 2011; 14: 101-112.

9. Washington K. 7th edition of the ajcc cancer staging manual: Stomach. Ann Surg Oncol 2010; 17: 3077-9.

10. Luo M, Lv Y, Guo X, Song H, Su G, Chen B. Value and impact factors of multidetector computed tomography in diagnosis of preoperative lymph node metastasis in gastric cancer: A PRISMA-compliant systematic review and meta-analysis. Medicine (Baltimore) 2017; 96: e7769.

11. Fukuya T, Honda H, Hayashi T, et al. Lymph-node metastases: Efficacy of detection with helical ct in patients with gastric cancer. Radiology 1995; 197: 705-711.

12. Morgagni P, Petrella E, Basile B, et al. Preoperative multidetector-row computed tomography scan staging for lymphatic gastric cancer spread. World J Surg Oncol 2012; 10: 197.

13. Kim DJ, Kim W. Is lymph node size a reliable factor for estimating lymph node metastasis in early gastric cancer? J Gastric Cancer 2018; 18: 20-29.

14. Yan C, Zhu ZG, Yan M, et al. Size of the largest lymph node visualized on multi-detector-row computed tomography (MDCT) is useful in predicting metastatic lymph node status of gastric cancer. $J$ Int Med Res 2010; 38: 22-33.

15. Ahn HS, Lee HJ, Yoo MW, et al. Diagnostic accuracy of T and N stages with endoscopy, stomach protocol CT, and endoscopic ultrasonography in early gastric cancer. J Surg Oncol 2009; 99: 20-27. 
16. Karakoyun R, Demirci E, Karakoyun M, et al. Reliability of MDCT, with MPR and hydro-CT technique, in resectability and lymphnode staging of gastric cancer. Minerva Chir 2014; 69: 129-140.

17. Feng XY, Wang W, Luo GY, et al. Comparison of endoscopic ultrasonography and multislice spiral computed tomography for the preoperative staging of gastric cancer - results of a single institution study of 610 chinese patients. PLoS One 2013; 8: e78846.

18. Pan Z, Pang L, Ding B, et al. Gastric cancer staging with dual energy spectral CT imaging. PLoS One 2013; 8: e53651.

19. Joo I, Lee JM, Kim JH, Shin Cl, Han JK, Choi BI. Prospective comparison of 3T MRI with diffusion-weighted imaging and MDCT for the preoperative TNM staging of gastric cancer. J Magn Reson Imaging 2015; 41: 814-821.

20. Bai H, Deng J, Zhang N, et al. Predictive values of multidetector-row computed tomography combined with serum tumor biomarkers in preoperative lymph node metastasis of gastric cancer. Chin J Cancer Res 2019; 31: 453-462.

21. Fujikawa H, Yoshikawa T, Hasegawa S, et al. Diagnostic value of computed tomography for staging of clinical T1 gastric cancer. Ann Surg Oncol 2014; 21: 3002-3007.

22. Yoshikawa T, Tanabe K, Nishikawa K, et al. Accuracy of CT staging of locally advanced gastric cancer after neoadjuvant chemotherapy: cohort evaluation within a randomized phase II study. Ann Surg Oncol 2014; 21: S385-389.

23. Yan C, Zhu ZG, Yan M, et al. Value of multidetector-row computed tomography in the preoperative $\mathrm{T}$ and $\mathrm{N}$ staging of gastric carcinoma: a large-scale Chinese study. J Surg Oncol 2009; 100: 205-214.

24. Kwee RM, Kwee TC. Imaging in assessing lymph node status in gastric cancer. Gastric Cancer 2009; 12: 6-22.

25. Gotoda T, Yanagisawa A, Sasako M, et al. Incidence of lymph node metastasis from early gastric cancer: estimation with a large num ber of cases at two large centers. Gastric Cancer 2000; 3: 219-225.

\section{Address for correspondence}

\section{Nguyen Minh Duc}

Department of Radiology

Pham Ngoc Thach University of Medicine

02 Duong Quang Trung St.

Ward 12, District 10

700000 Ho Chi Minh City, Vietnam

e-mail: bsnguyenminhduc@pnt.edu.vn

Submitted: 21.05 .2020

Accepted: 7.06.2020 